\title{
Dislocation Characterization using Weak Beam Dark Field STEM Imaging
}

\author{
J. Miao ${ }^{1,2}$, S. Singh ${ }^{3}$, J. Tessmer ${ }^{3}$, M. Shih ${ }^{2}$, M. Ghazisaeidi², M. DeGraef ${ }^{3}$, and M. J. Mills ${ }^{1,2}$ \\ 1. Center for Electron Microscopy and Analysis, The Ohio State University, Columbus, OH. \\ 2. Department of Materials Science and Engineering, The Ohio State University, Columbus, OH. \\ 3. Department of Materials Science and Engineering, Carnegie Mellon University, Pittsburgh, PA.
}

Dislocation characterization plays a critical role in understanding deformation behavior of engineering alloys. Most previous work in the field of dislocation analysis was conducted using Conventional Transmission Electron Microscopy (CTEM) in parallel beam condition. Recent study shows that similar to CTEM mode, dislocation imaging such as $g \cdot b$ visibility analysis also can be conducted in Scanning Transmission Electron Microscopy (STEM) [1]. Figure 1a) and 1b) shows illustrations of overall configuration of STEM imaging and STEM defect analysis using systematic row diffraction respectively. As compared with CTEM, STEM defect imaging provides additional advantages such as suppressing bend contours and being able to image thicker TEM specimens.

In CTEM mode, weak-beam imaging offers a powerful tool for studying fine dislocation structures such as dissociated dislocations [2]. In STEM mode, defect imaging using high order reflections can be achieved through tilting specimens to let the center of the high order diffraction disc, such as $3 \mathrm{~g}$ in a systematic row diffraction pattern, satisfy the Bragg condition. Under such a diffraction condition, either the bright-field disc or $3 \mathrm{~g}$ disc can be used for dislocation imaging [1]. In a recent study [3], dislocation imaging using the $1 \mathrm{~g}$ disc combined with a centered bright field detector was used. Such an imaging mode using the centered bright field detector is greatly limited by the available beam convergence angles and camera lengths. Uncertainty in interpreting results is also associated with this method when the transmitted and diffracted discs are overlapped in STEM mode.

In this study, a new imaging mode using the $1 \mathrm{~g}$ disc combined with diffraction apertures and annular dark field STEM detectors are used to achieve Weak Beam Dark Field STEM (WBDF STEM) modes. In this method, samples are tilted to make the $3 \mathrm{~g}$ disc satisfy the Bragg diffraction condition. A diffraction aperture is then used to select the $1 \mathrm{~g}$ disc for dark field imaging, and an annular dark field detector is used to collect diffraction signals. Under such an imaging mode, the above limitations and uncertainties can be excluded.

Figure 2 show an example of the application of the new WBDF STEM imaging method in studying dislocation dissociation in an equiatomic NiCoCr solid solution alloy. The sample was deformed to a true strain level of 5\% at cryogenic temperature. The details of the testing material and microstructure characterization can be found elsewhere [4]. Both WBDF STEM imaging and WB CTEM imaging were conducted on a FEI Tecnai F20 S/TEM operating at 200keV. Figure 2 shows the comparison of two imaging modes. As shown in Figure $2 \mathrm{c}$ ), the bend contours in CTEM mode in Figure 2 a) were completely suppressed in STEM mode. Three examples of measurement of dissociation distances are given both in WB CTEM image (Figure $2 \mathrm{~b}$ ) and WBDF STEM image (Figure $2 \mathrm{~d}$ ). It can be seen that WBDF STEM mode yields nearly the same results as those obtained from the WB CTEM method. Simulations of higher order $\mathrm{g}$ and WBDF STEM contrast will also be discussed and compared with experimental results [5]. 


\section{References:}

[1] P.J. Phillips, M.J. Mills, and M. De Graef, Philosophical Magazine 91 (2011), p. 2081.

[2] D.J.H. Cockayne, I. L.F. Ray and M. J. Whelan, Philosophical Magazine A 20 (1969), p. 1265.

[3] H. Iwata and H. Saka, Philosophical Magazine Letters 97 (2017), p. 74.

[4] J. Miao et al, Acta Materialia 132 (2017), p. 35.

[5] The authors acknowledge financial supports from The National Science Foundation, Division of Materials Research under contract \# \#DMR-60050072.

a

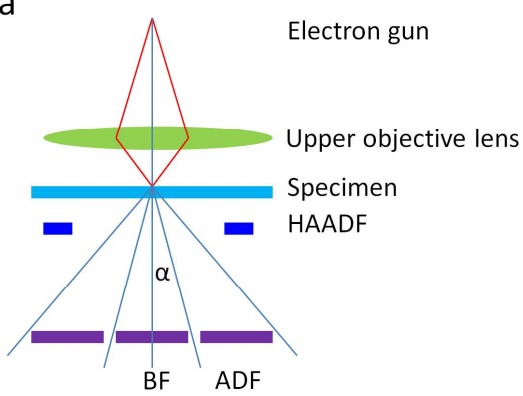

b

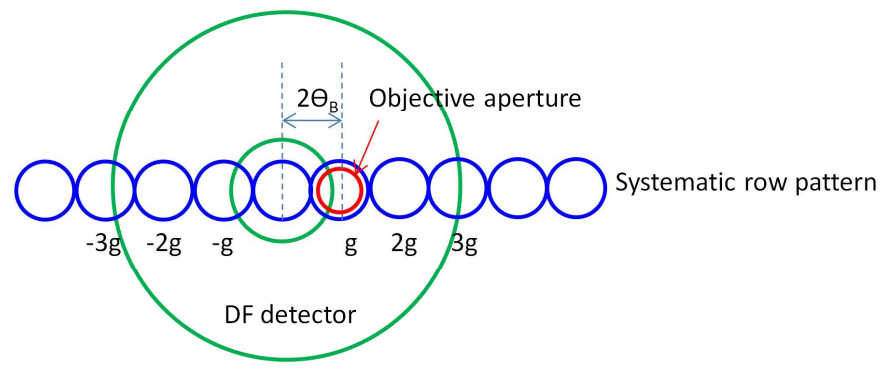

Figure 1. a) Schematic illustration the overall configuration of STEM imaging, and

b) schematic illustration of STEM imaging using systematic row diffraction with an diffraction aperture.
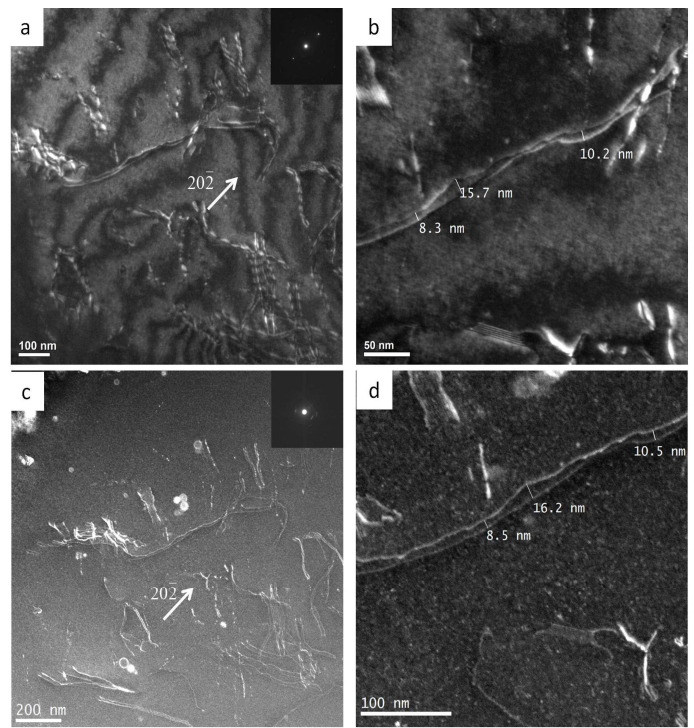

Figure 2. Comparison between WB CTEM imaging and WBDF STEM imaging of dissociated dislocations: a) low magnification WB CTEM image, b) high magnification WB CTEM image, c) low magnification WBDF STEM image, and d) high magnification WBDF STEM image. 